\title{
REGULARITY OF OPTIMAL TRANSPORTATION BETWEEN SPACES WITH DIFFERENT DIMENSIONS
}

\author{
BREndAN PASS
}

\begin{abstract}
We study the regularity of solutions to an optimal transportation problem in which the dimension of the source is larger than that of the target. We prove that, unless the cost $c$ has a very special form, (in which case we show that the problem can be reduced to an optimal transportation problem between equal dimensional spaces), there are smooth marginals for which the optimal map is discontinuous. If $c$ does not have this special form, we identify sufficient conditions on the cost and the marginals to ensure that the optimal map is continuous, in the case where the target is one dimensional.
\end{abstract}

\section{Introduction}

Let $X$ and $Y$ be connected smooth manifolds of dimensions $m$ and $n$, endowed with Borel probability measures $\mu$ and $\nu$, respectively. We say that a Borel map $F: X \rightarrow Y$ pushes $\mu$ forward to $\nu$ if for all Borel sets $A \subseteq Y$ we have $\nu(A)=\mu\left(F^{-1}(A)\right)$. For a given cost function $c: X \times Y \rightarrow \mathbb{R}$, Monge's optimal transportation problem is then to find the Borel map $F$ pushing $\mu$ forward to $\nu$ that minimizes the total transportation cost:

$$
\int_{X} c(x, F(x)) d \mu .
$$

This can be viewed as a stricter version of the Kantorovich optimal transportation problem, which is to minimize

$$
\int_{X \times Y} c(x, y) d \gamma
$$

among all Borel probability measures $\gamma$ on $X \times Y$ such that the projections of $X \times Y$ onto $X$ and $Y$ push $\gamma$ forward to $\mu$ and $\nu$, respectively. In fact, the usual method for finding solutions to Monge's problem is to first find the Kantorovich solution; one can then show that, under certain conditions, the solution $\gamma$ is concentrated on the graph of a function $F: X \rightarrow Y[2,6,16,17,21]$. These conditions cannot generally hold if $m<n$; in this case, however, there are known conditions under which $\gamma$ will concentrate on the graph of a function $H: Y \rightarrow X$ and so it is preferable to reformulate Monge's problem in terms of maps from $Y$ to $X$. When minimizing (1.1), then, it is natural to restrict our attention to the case when $m \geq n$.

Monge's problem has numerous applications and has received a lot of attention from many different authors. Questions about the existence and uniqueness of optimal maps have been resolved for a wide class of cost functions; much of the present research in optimal transportation aims to understand the structure of these optimizers. A great deal of progress has been made in this direction, but it has mostly

Received by the editors June 20, 2011. 
been restricted to the case when $m=n$. Problems where $m>n$, on the other hand, have received very little attention; the only results we are aware of in this direction concern the local rectifiability of the solution $[29,30]$. Aside from being a natural mathematical generalization of the relatively well understood $m=n$ case, however, optimal transportation problems where $m$ and $n$ fail to coincide may have important applications; for example, in economics, optimal transportation type problems arise frequently and there is often no compelling reason to assume that $m=n$. For a treatment of a related problem in an economic context, see [1] and [10]; the connections between these results and the present work are explored by the present author in a separate paper [31].

In the $m=n$ case, understanding the regularity, or smoothness, of the optimal map, has grown into an active and exciting area of research in the past few years, due to a major breakthrough by Ma et al. [26]. They identified a fourth-order differential condition on $c$ (called (A3s) in the literature) which implies the smoothness of the optimizer, provided the marginals $\mu$ and $\nu$ are smooth. Subsequent investigations by Trudinger and Wang $[33,34]$ revealed that these results actually hold under a slight weakening of this condition, called (A3w), encompassing earlier results of Caffarelli [3-5], Urbas [35] and Delanoe [8,9] when $c$ is the distance squared on either $\mathbb{R}^{n}$ or certain Riemannian manifolds and Wang for another special cost function [36]. Loeper [23] then verified that (A3w) is in fact necessary for the solution to be continuous for arbitrary smooth marginals $\mu$ and $\nu$. Loeper also proved that, under (A3w), the optimizer is Holder continuous even for rougher marginals; this result was subsequently improved by Liu [22], who found a sharp Holder exponent. Since then, many interesting results about the regularity of optimal transportation have been established [11-15, 18-20,24,25].

This article focuses on adapting these results to the $m>n$ setting. A serious obstacle arises immediately; the regularity theory of Ma et al. requires invertibility of the matrix of mixed second-order partials $\left(\frac{\partial^{2} c}{\partial x^{i} \partial y^{j}}\right)_{i j}$, and its inverse appears explicitly in their formulations of (A3w) and (A3s). When $m$ and $n$ fail to coincide, however, $\left(\frac{\partial^{2} c}{\partial x^{i} \partial y^{j}}\right)_{i j}$ clearly cannot be invertible. Alternate formulation of $(\mathbf{A} \mathbf{3 w})$ and $(\mathbf{A} \mathbf{3 s})$ that do not explicitly use this invertibility were developed by Loeper (see [23], Section 2); however, they rely instead on local surjectivity of the map $y \mapsto D_{x} c(x, y)$, which cannot hold in our setting either.

Nonetheless, there is a certain class of costs for which our problem can easily be solved using the results from the equal dimensional setting. Suppose $c(x, y)=$ $b(Q(x), y)$, where $Q: X \rightarrow Z$ is smooth and $Z$ is a smooth manifold of dimension $n$; we will call such costs reducible. In this case, we show that the optimal map takes every point in each level set of $Q$ to a common $y$ and studying its regularity amounts to studying an optimal transportation problem on the $n$-dimensional spaces $Z$ and $Y$. More surprisingly, we will show that reducible costs are essentially the only costs on $X \times Y$ for which we can hope for regularity results for arbitrary smooth marginals $\mu$ and $\nu$. Indeed, for the quadratic cost on Euclidean domains, the regularity theory of Caffarelli requires convexity of the target $Y[3,4]$ and, for general costs, it became apparent in the work of Ma et al. [26] that continuity of the optimizer cannot hold for arbitrary smooth marginals unless $Y$ satisfies an appropriate, generalized notion of convexity. Due to its dependence on the cost function, this condition is referred to 
as $c$-convexity; when $m>n$, we will show that $c$-convexity necessarily fails unless the cost function is of the reducible form alluded to above.

In the next section, we will introduce preliminary concepts from the regularity theory of optimal transportation, suitably adapted for general values of $m \geq n$. In the third section, we prove that if $c$ is not reducible, then there are smooth measures for which the optimal map is discontinuous. For reducible costs, we then show that the properties on $Z$ and $b$ which are necessary and sufficient for the optimal map to be continuous follow from analogous properties on $X$ and $c$.

Given the preceding discussion, it is apparent that for cost functions that are not reducible, there are smooth marginals for which the optimal map is discontinuous. However, as the reducibility condition is so restrictive, it is natural to ask about regularity for costs which are not of this form; any result in this direction will require stronger conditions on the marginals than smoothness. In the final section of our paper, we address this problem when $n=1$.

Finally, let us mention that the calculations in Section 2 of a recent paper by Castillon [7] are somewhat similar to some of those found here, which was pointed out to us by an anonymous referee after this article had been submitted. In [7], $c$ is equal to the distance squared on Euclidean space, and the target measure is supported on a lower dimensional affine subspace; in our terminology, this cost function is reducible.

\section{Conditions and definitions}

Here, we develop several definitions and conditions which we will require in the following sections. We begin with some basic notation. In what follows, we will assume that $X$ and $Y$ may be smoothly embedded in larger manifolds in which their closures, $\bar{X}$ and $\bar{Y}$, are compact. If $c$ is differentiable, we will denote by $D_{x} c(x, y)$ its differential with respect to $x$. If $c$ is twice differentiable, $D_{x y}^{2} c(x, y)$ will denote the map from the tangent space of $Y$ at $y, T_{y} Y$, to the cotangent space of $X$ at $x, T_{x}^{*} X$, defined in local coordinates by

$$
\frac{\partial}{\partial y^{i}} \mapsto \frac{\partial^{2} c(x, y)}{\partial y^{i} \partial x^{j}} d x^{j},
$$

where summation on $j$ is implicit, in accordance with the Einstein summation convention. $D_{y} c(x, y)$ and $D_{y x}^{2} c(x, y)$ are defined analogously.

A function $u: X \rightarrow \mathbb{R}^{n}$ is called $c$-concave if $u(x)=\inf _{y \in Y} c(x, y)-u^{c}(y)$, where $u^{c}(y):=\inf _{x \in X} c(x, y)-u(x)$.

Next, we recall the concept of $c$-convexity, which first appeared in [26].

Definition 2.1. We say domain $Y$ looks $c$-convex from $x \in X$ if $D_{x} c(x, Y)=$ $\left\{D_{x} c(x, y) \mid y \in Y\right\}$ is a convex subset of $T_{x} X$. We say $Y$ is $c$-convex with respect to $X$ if it looks $c$-convex from every $x \in X$.

Our next definition is novel, as it is completely irrelevant when $m=n$. It will, however, play a vital role in the present setting.

Definition 2.2. We say domain $Y$ looks $c$-linear from $x \in X$ if $D_{x} c(x, Y)$ is contained in a shifted, $n$-dimensional, linear subspace of $T_{x} X$. We say $Y$ is $c$-linear with respect to $X$ if it looks $c$-linear from every $x \in X$. 
When $m=n, c$-linearity is automatically satisfied. When $m>n$, this is no longer true, although $c$-convexity clearly implies $c$-linearity.

We will also have reason to consider the level set of $\bar{x} \mapsto D_{y} c(\bar{x}, y)$ passing through $x, L_{x}(y):=\left\{\bar{x} \in X: D_{y} c(\bar{x}, y)=D_{y} c(x, y)\right\}$.

Let us now state our first three regularity conditions. When $m=n$, these are identical to the conditions (A0)-(A2) introduced by Ma et al.

(A0'): The function $c \in C^{4}(\bar{X} \times \bar{Y})$.

(A1'): (Twist) For all $x \in X$, the map $y \mapsto D_{x} c(x, y)$ is injective on $\bar{Y}$.

(A2'): (Non-degeneracy) For all $x \in X$ and $y \in Y$, the map $D_{x y}^{2} c(x, y): T_{y} Y \rightarrow$ $T_{x}^{*} X$ is injective.

Remark 2.1. When $m=n$, a bi-twist hypothesis is required to prove regularity of the optimal map; in addition to (A1'), one must assume $x \mapsto D_{y} c(x, y)$ is injective on $X$ for all $y \in Y$. Clearly, such a condition cannot hold if $m>n$; in fact, the non-degeneracy condition and the implicit function theorem imply that the level sets $L_{x}(y)$ of this mapping are smooth $m-n$-dimensional hypersurfaces. Later, we will assume that the these level sets are connected. When $m=n$, non-degeneracy implies that each $L_{x}(y)$ consists of finitely many isolated points, in which case connectedness implies that it is in fact a singleton, or, equivalently, that $x \mapsto D_{y} c(x, y)$ is injective.

When $m=n$, examples of non-degenerate costs for which the bi-twist condition fails (in which case the level sets of $x \mapsto D_{y} c(x, y)$ are not connected) can be found in [28], Examples 3.1 and 3.2.

The statements of (A3w') and (A3s'), the most important regularity conditions, require a little more machinery. For a twisted cost, the mapping $y \mapsto D_{x} c(x, y)$ is invertible on its range. We define the $c$-exponential map at $x$, denoted by $c$ - $\exp _{x}(\cdot)$, to be its inverse; that is, $D_{x} c\left(x, c-\exp _{x}(p)\right)=p$ for all $p \in D_{x} c(x, Y)$.

Next, we define the Ma-Trudinger-Wang curvature. Note that our formulation requires $c$-linearity.

Definition 2.3. Assume $Y$ is $c$-linear and that (A0')-(A2') hold. Let $x \in X$ and $y \in Y$. Choose tangent vectors $\mathbf{u} \in T_{x} X$ and $\mathbf{v} \in T_{y} Y$. Set $\mathbf{p}=D_{x} c(x, y) \in T_{x}^{*} X$ and $\mathbf{q}=\left(D_{x y}^{2} c(x, y)\right) \cdot \mathbf{v} \in T_{x}^{*} X$; note that as $Y$ looks $c$-linear at $x, \mathbf{p}+t \mathbf{q} \in D_{x} c(x, Y)$ for small $t$. For any smooth curve $\beta(s)$ in $X$ with $\beta(0)=x$ and $\frac{d \beta}{d s}(0)=\mathbf{u}$, we define the Ma-Trudinger-Wang curvature at $x$ and $y$ in the directions $\mathbf{u}$ and $\mathbf{v}$ by

$$
\operatorname{MTW}_{x y}\langle\mathbf{u}, \mathbf{v}\rangle:=-\frac{3}{2} \frac{\partial^{4}}{\partial s^{2} \partial t^{2}} c\left(\beta(s), c-\exp _{x}(\mathbf{p}+t \mathbf{q})\right) \text {. }
$$

When $m=n$, Loeper showed that $\mathrm{MTW}_{x y}$ is invariant under changes of coordinates and demonstrated that, for the quadratic cost on a Riemannian manifold, it coincides with sectional curvature on the diagonal [23]. Kim and McCann then showed that it is actually the sectional curvature arising from a certain pseudo-metric on $X \times Y$ [19]. Although our definition here is new for $m>n$, it coincides with the definitions in [23] and [19] when $m=n$.

We are now ready to state the final conditions of Ma et al. Because they are designed to deal with the general case $m \geq n$, our formulations look somewhat different from the conditions (A3w) and (A3s) found in [26]; when $m=n,\left(\mathbf{A 3} \mathbf{w}^{\prime}\right)$ and (A3s') reduce to $(\mathbf{A} 3 \mathbf{w})$ and $(\mathbf{A} 3 \mathbf{s})$, respectively. Unlike (A0')-(A2'), the formulation of these conditions requires $c$-linearity, as they involve the Ma-Trudinger-Wang curvature. 
(A3w'): For all $x \in X, y \in Y, \mathbf{u} \in T_{x} X$ and $\mathbf{v} \in T_{y} Y$ such that $\mathbf{u} \cdot D_{x y}^{2} c(x, y) \cdot \mathbf{v}=0$, $\mathrm{MTW}_{x y}\langle\mathbf{u}, \mathbf{v}\rangle \geq 0$.

(A3s'): For all $x \in X, y \in Y, \mathbf{u} \in T_{x} X$ and $\mathbf{v} \in T_{y} Y$ such that $\mathbf{u} \cdot\left(D_{x y}^{2} c(x, y)\right) \cdot \mathbf{v}=$ $0, \mathbf{u} \cdot\left(D_{x y}^{2} c(x, y)\right) \neq 0$ and $\mathbf{v} \neq 0$ we have $\mathrm{MTW}_{x y}\langle\mathbf{u}, \mathbf{v}\rangle>0$.

Note that, if $m=n$, non-degeneracy implies that the condition $\mathbf{u} \cdot\left(D_{x y}^{2} c(x, y)\right) \neq 0$ is equivalent to $\mathbf{u} \neq 0$.

Finally, we define the notion of a reducible cost function, alluded to in the introduction.

Definition 2.4. A cost function $c$ is called reducible if there exists a smooth $n$ dimensional manifold $Z$, a smooth map $Q: X \rightarrow Z$, a function $b: Z \times Y \rightarrow \mathbb{R}$ and a map $a: X \rightarrow \mathbb{R}$ such that, for all $(x, y) \in X \times Y$

$$
c(x, y)=b(Q(x), y)+a(x) .
$$

\section{Regularity of optimal maps}

The following theorem asserts the existence of an optimal map. It is due to Levin [21] in the case where $X$ is a bounded domain in $\mathbb{R}^{m}$ and $\mu$ is absolutely continuous with respect to Lebesgue measure. The following version can be proved in the same way; see also Brenier [2], Gangbo [16], Gangbo and McCann [17] and Caffarelli [6] and McCann [27].

Theorem 3.1. Suppose $c$ is twisted and $\mu(A)=0$ for all Borel sets $A \subseteq X$ of Hausdorff dimension less than or equal to $m-1$. Then the Monge problem admits a unique solution $F$ of the form $F(x)=c$-exp $(x, D u(x))$ for some c-concave function $u$.

The purpose of this section is to understand the smoothness of the optimal map $F$.

3.1. Discontinuity of optimal maps for non-reducible costs. This subsection is devoted to the proof of the following result.

Theorem 3.2. Assume that the level sets $L_{x}(y)$ are all connected. If $c$ is not reducible, there exist smooth marginals $\mu$ and $\nu$ for which the optimal map is discontinuous.

Our proof relies on the following result, which confirms the necessity of $c$-convexity to regularity. It is due to Ma et al. in the case where $m=n$ (see [26], Section 7.3); their proof applies to the $m \geq n$ case as well.

Theorem 3.3. Suppose there exists some $x \in X$ such that $Y$ does not look c-convex from $x$. Then there exist smooth measures $\mu$ and $\nu$ for which the optimal map is discontinuous.

As $c$-convexity implies $c$-linearity, this example verifies that we cannot hope to develop a regularity theory in the absence of $c$-linearity. Proposition 3.1 below demonstrates that, under the $c$-linearity hypothesis, the level sets $L_{x}(y)$ are the same for each $y$, yielding a canonical foliation of the space $X$, and the cost is reducible. To prove Proposition 3.1, we will need the following lemma.

Lemma 3.1. The domain $Y$ looks c-linear from $x \in X$ if and only if $T_{x}\left(L_{x}(y)\right)$ is independent of $y$; that is $T_{x}\left(L_{x}\left(y_{0}\right)\right)=T_{x}\left(L_{x}\left(y_{1}\right)\right)$ for all $y_{0}, y_{1} \in Y$. 
Proof. The tangent space to $L_{x}(y)$ at $x$ is the null space of the map $D_{y x}^{2} c(x, y)$ : $T_{x} X \mapsto T_{y}^{*} Y$, which, in turn, is the orthogonal complement of the range of $D_{x y}^{2} c(x, y)$ : $T_{y} Y \mapsto T_{x}^{*} X$. Therefore, $T_{x}\left(L_{x}(y)\right)$ is independent of $y$ if and only if the range of $D_{x y}^{2} c(x, y)$ is independent of $y$. But $D_{x y}^{2} c(x, y)$ is the differential of the map $y \mapsto$ $D_{x} c(x, y)$ (making the obvious identification between $T_{x}^{*} X$ and its tangent space at a point) and so its range is independent of $y$ if and only if the image of this map is linear.

Proposition 3.1. Suppose that the level sets $L_{x}(y)$ are all connected. Then the following are equivalent:

(1) $Y$ is c-linear with respect to $X$.

(2) $L_{x}(y)$ is independent of $y$ for all $x$; in this case we denote it simply by $L_{x}$.

(3) $c(x, y)=b(Q(x), y)+a(x)$ is reducible and the mapping $z \mapsto D_{y} b(z, y)$ is injective.

Proof. We first prove the equivalence of (1) and (2). Note that Lemma 3.1 implies $Y$ is $c$-linear with respect to $X$ if and only if $T_{x}\left(L_{x}\left(y_{0}\right)\right)=T_{x}\left(L_{x}\left(y_{1}\right)\right)$ for all $x \in X$ and all $y_{0}, y_{1} \in Y$. But $T_{x}\left(L_{x}\left(y_{0}\right)\right)=T_{x}\left(L_{x}\left(y_{1}\right)\right)$ for all $x$ is equivalent to $L_{x}\left(y_{0}\right)=L_{x}\left(y_{1}\right)$ for all $x$; this immediately yields the equivalence of (1) and (2).

To see that (3) implies (2), note that if $c(x, y)=b(Q(x), y)+a(x)$, the injectivity of $z \mapsto D_{y} b(z, y)$ implies that the level sets of $x \mapsto D_{y} c(x, y)=D_{y} b(Q(x), y)$ are precisely the level sets of $x \mapsto Q(x)$, which are inherently independent of $y$.

It remains to show that (2) implies (3). Assuming that the sets $L_{x}(y)$ are independent of $y$, we define an equivalence relation on $X$ by $x \sim \bar{x}$ if $\bar{x} \in L_{x}$. We then define the quotient space $Z=X / \sim$ and the quotient map $Q: X \rightarrow Z$. Note that, for any fixed $y_{0} \in Y$, the map $x \mapsto D_{y} c\left(x, y_{0}\right) \in T_{y_{0}}^{*} Y$ has the same level sets as $Q$ (namely the $L_{x}$ 's) and is smooth by assumption. Furthermore, the non-degeneracy condition implies that this map is open and hence a quotient map. We can therefore identify $Z \approx D_{y} c\left(X, y_{0}\right)$ with a subset of the cotangent space $T_{y_{0}}^{*} Y$. In particular, $Z$ has a smooth structure, and, if $c$ satisfies (A0'), $Q$ is $C^{3}$.

We define $b: Z \times Y \rightarrow \mathbb{R}^{n}$ by

$$
b(z, y)=c(x, y)-c\left(x, y_{0}\right)
$$

for any $x \in Q^{-1}(z)$. We must show that this is well defined; that is, for any $x, \bar{x} \in$ $Q^{-1}(z)$, we must show that $c(x, y)-c\left(x, y_{0}\right)=c(\bar{x}, y)-c\left(\bar{x}, y_{0}\right)$, or

$$
B(y):=c(x, y)-c\left(x, y_{0}\right)-c(\bar{x}, y)+c\left(\bar{x}, y_{0}\right)=0,
$$

for all $y \in Y$. Note that $x, \bar{x} \in Q^{-1}(z)$ means that $\bar{x} \in L x$, or

$$
D_{y} c(x, y)=D_{y} c(\bar{x}, y) \text {, }
$$

for all $y$. Now, (3.2) clearly holds at $y=y_{0}$. As (3.3) implies that $D_{y} B(y)=0$ for all $y$, it now follows that $B(y)=0$ for all $y$, as desired.

Now, our definition of $b$ immediately implies that $c(x, y)=b(Q(x), y)+c\left(x, y_{0}\right)$, i.e., $c$ is reducible.

We can now prove Theorem 3.2; the argument is a simple application of Proposition 3.1, together with Theorem 3.3 of Ma et al. 
Proof. Suppose $c$ is not reducible. Then Proposition 3.1 implies that $Y$ cannot be $c$-linear with respect to $X$. Theorem 3.3 then directly implies the desired result.

3.2. Regularity for reducible costs. Theorem 3.2 implies that we cannot hope to prove regularity results for arbitrary smooth marginals for non-reducible costs. For that reason, we focus in this section on reducible costs. We will show that, for a reducible cost, the optimal transportation problem can be transformed into a problem between two $n$-dimensional spaces; questions about the regularity of the optimal map can then be answered using the results of Ma et al. [26] and Loeper [23]. We also demonstrate that the relevant regularity conditions for this new problem are related to the conditions $\left(\mathbf{A} \mathbf{0}^{\prime}\right)-\left(\mathbf{A} \mathbf{3} \mathbf{s}^{\prime}\right)$.

For a reducible cost $c$, Proposition 3.1 implies that the level sets $L_{x}$ are independent of $y$; heuristically, points in the same level set are indistinguishable from an optimal transportation perspective. The $L_{x}$ 's define a canonical foliation of $X$ and in this subsection we show that our problem can be reduced to an optimal transportation problem between $Y$ and the space of leaves of this foliation. We will show that if $F: X \rightarrow Y$ is the optimal map, then $F$ factors through $Q ; F=T \circ Q$. As $Q$ is smooth, this will imply that treating the smoothness of $F$ reduces to studying the smoothness of $T$. To this end, we will show that $T$ itself solves an optimal transportation problem with marginals $\alpha=Q_{\#} \mu$ on $Z$ and $\nu$ on $Y$ relative to the cost function $b(z, y)$.

Lemma 3.1. For any $x_{0}, x_{1} \in L_{x}, \bar{y} \in Y$ and c-concave $u$ we have $u\left(x_{0}\right)=c\left(x_{0}, \bar{y}\right)-$ $u^{c}(\bar{y})$ if and only if $u\left(x_{1}\right)=c\left(x_{1}, \bar{y}\right)-u^{c}(\bar{y})$.

Proof. First note that as $D_{y} c\left(x_{0}, y\right)-D_{y} c\left(x_{1}, y\right)=0$ for all $y \in Y$, the difference $c\left(x_{0}, y\right)-c\left(x_{1}, y\right)$ is independent of $y$. Now, suppose $u\left(x_{0}\right)=c\left(x_{0}, \bar{y}\right)-u^{c}(\bar{y})$. Then

$$
\begin{aligned}
u\left(x_{1}\right) & =\inf _{y \in Y} c\left(x_{1}, y\right)-u^{c}(y) \\
& =\inf _{y \in Y}\left(c\left(x_{1}, y\right)-c\left(x_{0}, y\right)+c\left(x_{0}, y\right)-u^{c}(y)\right) \\
& =c\left(x_{1}, \bar{y}\right)-c\left(x_{0}, \bar{y}\right)+\inf _{y \in Y}\left(c\left(x_{0}, y\right)-u^{c}(y)\right) \\
& =c\left(x_{1}, \bar{y}\right)-c\left(x_{0}, \bar{y}\right)+u\left(x_{0}\right) \\
& =c\left(x_{1}, \bar{y}\right)-u^{c}(\bar{y}) .
\end{aligned}
$$

The proof of the converse is identical.

Lemma 3.2. Suppose $c$ is twisted and $\mu$ does not charge sets of Hausdorff dimension $m-1$. Let $F: X \rightarrow Y$ be the optimal map. Then there exists a map $T: Z \rightarrow Y$ such that $F=T \circ Q, \mu$ almost everywhere. Moreover, $T$ solves the optimal transportation problem on $Z \times Y$ with cost function $b$ and marginals $\alpha$ and $\nu$.

Proof. It is well known that there exists a $c$-concave function $u(x)$ such that, for $\mu$ almost every $x$, there is a unique $y \in Y$ such that $u(x)=c(x, y)-u^{c}(y)$; in this case, $F(x)=y$.

For $\alpha$ almost every $z \in Z$, Lemma 3.1 now implies that there is a unique $y \in Y$ such that $u(x)=c(x, y)-u^{c}(y)$ for all $x \in Q^{-1}(z)$; define $T(z)$ to be this $y$. In then follows immediately that $F=T \circ Q, \mu$ almost everywhere, and that $T$ pushes $\alpha$ to $\nu$. 
Now, suppose $G: Z \rightarrow Y$ is another map pushing $\alpha$ to $\nu$. Then $G \circ Q$ pushes $\mu$ to $\nu$ and because of the optimality of $F=T \circ Q$ we have

$$
\int_{X} c(x, T \circ Q(x)) d \mu \leq \int_{X} c(x, G \circ Q(x)) d \mu .
$$

Now, using (3.1) we have

$$
\begin{aligned}
\int_{X} c(x, T \circ Q(x)) d \mu & =\int_{X} b(Q(x), T \circ Q(x))+c\left(x, y_{0}\right) d \mu \\
& =\int_{Z} b(z, T(z)) d \alpha+\int_{X} c\left(x, y_{0}\right) d \mu .
\end{aligned}
$$

Similarly,

$$
\int_{X} c(x, G \circ Q(x)) d \mu=\int_{Z} b(z, G(z)) d \alpha+\int_{X} c\left(x, y_{0}\right) d \mu
$$

and so (3.4) becomes

$$
\int_{Z} b(z, T(z)) d \alpha \leq \int_{Z} c(z, G(z)) d \alpha
$$

Hence, $T$ is optimal.

Having established that the optimal map $F$ from $X$ to $Y$ factors through $Z$ via the quotient $Q$ and the optimal map $T$ from $Z$ to $Y$, we will now study how the regularity conditions (A1') - (A3s') for $c$ translate to $b$.

Equation (3.1) allows us to understand the derivatives of $b$ with respect to $z$. Pick a point $z_{0} \in Z$ and select $x_{0} \in Q^{-1}\left(z_{0}\right)$. Now, let $S$ be an $n$-dimensional surface passing through $x_{0}$ which intersects $L_{x_{0}}$ transversely. As the null space of the map $D_{y x}^{2} c\left(x, y_{0}\right): T_{x} X \rightarrow T_{y}^{*} Y$ is precisely $T_{x} L_{x}$ for any $y$, it is invertible when restricted to $T_{x} S$; by the inverse function theorem, the map $D_{y} c\left(\cdot, y_{0}\right)$ restricts to a local diffeomorphism on $S$. For all $z$ near $z_{0}$, there is a unique $x \in S \cap Q^{-1}(z)$ and we have $b(z, y)=$ $c(x, y)-c\left(x, y_{0}\right)$; we can now identify $\left.D_{z} b(z, y) \approx D_{x} c\right|_{S \times Y}(x, y)-\left.D_{x} c\right|_{S \times Y}\left(x, y_{0}\right)$ and $\left.D_{z y}^{2} b(z, y) \approx D_{x y}^{2} c\right|_{S \times Y}(x, y)$. We use this observation to prove the following result.

Theorem 3.4. Assume that $c$ is reducible and the level sets $L_{x}$ are connected. Then

(1) If $c$ is twisted, $b$ is bi-twisted.

(2) If $c$ is non-degenerate, $b$ is non-degenerate.

(3) If $Y$ is c-convex, it is also b-convex.

Proof. Assuming the twistedness of $c$, we first prove the injectivity of $z \mapsto D_{y} b(x, y)$; suppose $D_{y} b\left(z_{0}, y\right)=D_{y} b\left(z_{1}, y\right)$. By $(3.1)$ we have $D_{y} b\left(z_{i}, y\right)=D_{y} c\left(x_{i}, y\right)$ for $x_{i} \in$ $Q^{-1}\left(z_{i}\right), i=0,1$. Thus, $D_{y} c\left(x_{0}, y\right)=D_{y} c\left(x_{1}, y\right)$, which, as $L_{x}(y)=L_{x}\left(y_{0}\right)$ by Proposition 3.1, implies

$$
z_{0}=Q\left(x_{0}\right)=D_{y} c\left(x_{0}, y_{0}\right)=D_{y} c\left(x_{1}, y_{0}\right)=Q\left(x_{1}\right)=z_{1} .
$$

Injectivity of $y \mapsto D_{z} b(z, y)$ and follows from the preceding identification. Similarly, assuming the non-degeneracy of $c$, the non-degeneracy of $b$ follows from this identification as well.

Note that transversality implies $T_{x}^{*} X=T_{x}^{*} L_{x} \oplus T_{x}^{*} S$. Our local identification between $Z$ and $S$ identifies the projection of the range $D_{x} c(x, Y)$ onto $T_{x}^{*} S$ with 
$D_{z} b(z, Y)$. As the projection of a convex set is convex, the $b$-convexity of $Y$ now follows from its $c$-convexity.

Theorem 3.5. Assume that $c$ is reducible and the level sets $L_{x}$ are connected. Then the following are equivalent:

(1) b satisfies $(\boldsymbol{A} 3 \boldsymbol{w})$.

(2) c satisfies $\left(\boldsymbol{A} 3 \boldsymbol{w}^{\prime}\right)$.

(3) c satisfies ( $\boldsymbol{A} 3 \boldsymbol{w}^{\prime}$ ) when restricted to $S \times Y$, where $S \subseteq X$ is any smooth surface of dimension $n$ which is transverse to each $L_{x}$ that it intersects.

Proof. The equivalence of (1) and (3) follow immediately from our identification. Clearly, (2) implies (3); to see that (3) implies (2) it suffices to show $\operatorname{MTW}_{x y}\langle\mathbf{u}, \mathbf{v}\rangle=0$ when $\mathbf{u} \in T_{x} L_{x}$, as $M T W_{x y}$ is linear in $\mathbf{u}$. Choosing a curve $\beta(s) \in L_{x}$ such that $\beta(0)=x$ and $\frac{d \beta}{d s}(0)=\mathbf{u}$ and $\mathbf{p}, \mathbf{q}$ as in the definition, we have

$$
\frac{d \beta}{d s}(s) \in T_{\beta(s)} L_{\beta(s)}=\operatorname{null}\left(D_{x y}^{2} c\left(\beta(s), c-\exp _{x}(\mathbf{p}+t \mathbf{q})\right)\right),
$$

for all $s$ and $t$, yielding

$\frac{d^{2}}{d s d t} c\left(\beta(s), c-\exp _{x}(\mathbf{p}+t \mathbf{q})\right)=\frac{d \beta}{d s} \cdot D_{x y}^{2} c\left(\beta(s), c-\exp _{x}(\mathbf{p}+t \mathbf{q})\right) \cdot \frac{d(c-\exp (\mathbf{p}+t \mathbf{q}))}{d t}=0$ Hence, $\operatorname{MTW}_{x y}\langle\mathbf{u}, \mathbf{v}\rangle=0$.

Theorem 3.6. Assume that $c$ is reducible and the level sets $L_{x}$ are connected. Then the following are equivalent:

(1) b satisfies (A3s).

(2) c satisfies (A3s').

(3) c satisfies (A3s') when restricted to $S \times Y$, where $S \subseteq X$ is any smooth surface of dimension $m$ which is transverse to each $L_{x}$ that it intersects.

Proof. The equivalence follows immediately from the identification, after observing that the $v \cdot\left(D_{x y}^{2} c(x, y)\right) \neq 0$ condition in the definition of (A3s') excludes the nontransverse directions.

Various regularity results for $T$ (and therefore $F$ ) now follow from the regularity results of Ma et al. [26], Loeper [23] and Liu [22]. Note, however, that these results all require certain regularity hypotheses on the marginals; to apply them in the present context, we must check these conditions on $\alpha$, rather than $\mu$. A brief discussion on whether the relevant regularity conditions on $\mu$ translate to $\alpha$ therefore seems in order.

First, suppose $X$ is a bounded domain in $\mathbb{R}^{m}$ and $\mu=f(x) d x$ is absolutely continuous with respect to $m$-dimensional Lebesgue measure. Then $\alpha$ is absolutely continuous with respect to $n$-dimensional Lebesgue measure with density $h(z)$ given by the coarea formula:

$$
h(z):=\int_{Q^{-1}(z)} \frac{f(x)}{J Q(x)} d H^{m-n}(x),
$$

where $J Q$ is the Jacobian of the map $Q$, restricted to the orthogonal complement of $T_{x} L_{x}$.

Lemma 3.2. Suppose $f \in L^{p}(X)$ (with respect to Lebesgue measure on $X$ ) for some $p \in[1, \infty]$. Then $h \in L^{p}(Z)$. 
Proof. We have $h^{p}(z)=\left(\int_{Q^{-1}(z)} \frac{f(x)}{J Q(x)} d H^{m-n}(x)\right)^{p}$. By non-degeneracy, $J Q(x)>0$, for all $x \in \bar{X}$; by compactness, it has a positive global lower bound $K>0$. Normalizing and applying Jensen's inequality yields:

$$
\begin{aligned}
\frac{h^{p}(z)}{C^{p}(z)} & \leq \int_{Q^{-1}(z)} \frac{f^{p}(x)}{(J Q(x))^{p} C(z)} d H^{m-n}(x) \\
& \leq \int_{Q^{-1}(z)} \frac{f^{p}(x)}{J Q(x) C(z) K^{p-1}} d H^{m-n}(x),
\end{aligned}
$$

where $C(z)$ is the $(m-n)$-dimensional Hausdorff measure of $Q^{-1}(z)$. Letting $C$ be a global upper bound on $C(z)$ and integrating over $z$ implies

$$
\begin{aligned}
\int h^{p}(z) d z & \leq \iint_{Q^{-1}(z)} \frac{f^{p}(x) C^{p-1}(z)}{J Q(x)^{p} K^{p-1}} d H^{m-n}(x) d z \\
& \leq \frac{C^{p-1}}{K^{p-1}} \iint_{Q^{-1}(z)} \frac{f^{p}(x)}{J Q(x)^{p}} d H^{m-n}(x) d z \\
& =\frac{C^{p-1}}{K^{p-1}} \int f^{p}(x) d x<\infty
\end{aligned}
$$

where we have again used the coarea formula in the last step.

Let us note, however, that an analogous result does not hold for the weaker condition introduced by Loeper [23], which requires that for all $x \in X$ and $\epsilon>0$

$$
\mu\left(B_{\epsilon}(x)\right) \leq K \epsilon^{n\left(1-\frac{1}{p}\right)},
$$

for some $p>n$ and $K>0$. Indeed, if $m-n \geq n$, we can take $\mu$ to be $(m-n)$ dimensional Hausdorff measure on a single level set $L_{x}$. Then $\mu$ will satisfy the above condition for any $p$, but $\alpha$ will consist of a single Dirac mass.

The preceding lemma allows us to immediately translate the regularity results of Loeper and Liu to the present setting.

Corollary 3.1. Suppose that $Y$ is c-convex with connected level sets $L_{x}(y)$ for all $x \in X$ and $y \in Y$, and that (AO'), (A1'), (A2') and (A3s') hold. Suppose that $f \in L^{p}(X)$ for some $p>\frac{n+1}{2}$. Then the optimal map is Holder continuous with Holder exponent $\frac{\beta(n+1)}{2 n^{2}+\beta(n-1)}$, where $\beta=1-\frac{n+1}{2 p}$.

The higher regularity results of Ma et al. require $C^{2}$ smoothness of the density $h$. As the following example demonstrates, however, smoothness of $f$ does not even imply continuity of $h$.

Example 3.1. Let

$$
X=\left\{x=\left(x_{1}, x_{2}\right):-1<x_{1}<1,-1<x_{2}<\phi\left(x_{1}\right)\right\} \subseteq \mathbb{R}^{2},
$$

where $\phi:(-1,1) \rightarrow(-1,1)$ is a $C^{\infty}$ function such that $\phi\left(x_{1}\right)=0$ for all $-1<x_{1}<0$, $\phi(1)=1$ and $\phi$ is strictly increasing on $(0,1)$. Let $Y=(0,1) \subseteq \mathbb{R}$ and $c(x, y)=x_{2} y$. Then $Y$ is $c$-convex and $c$ satisfies (A0')-(A3s'). The level sets $L_{x}$ are simply the curves $\left\{x: x_{2}=c\right\}$ for constant values of $c \in(-1,1)$ and $Z=(-1,1)$. Set $f(x)=k$, where $k$ is a constant chosen so that $\mu$ has total mass 1 . The density $h$ is then easy 
to compute; it is simply the length of the line segment $Q^{-1}(z)$. For $z<0, h(z)=2 k$; however, for $z>0, h(z)=k\left(1-\phi^{-1}(z)\right)<k .^{1}$

On the other hand, we should note that is possible for $\alpha$ to be smooth even when $\mu$ is singular. This will be the case if, for example, $\mu$ is $n$-dimensional Hausdorff measure concentrated on some smooth $n$-dimensional surface $S$ which intersects the $L_{x}$ 's transversely.

Finally, we exploit Loeper's counterexample, which shows that, when $m=n$ and (A3w') fails, there are smooth densities for which the optimal map is not continuous.

Corollary 3.2. Suppose that $Y$ is c-convex and that the level sets $L_{x}(y)$ are connected for all $x \in X$ and $y \in Y$. Assume (AO'), (A1') and (A2') hold but (A3w') fails. Then there are smooth marginals $\mu$ on $X$ and $\nu$ on $Y$, with densities bounded above and below, such that the optimal map is discontinuous.

Proof. Using equation (3.1), it is easy to check that $u: X \rightarrow \mathbb{R}$ is $c$-concave if and only it $u(x)=v(Q(x))+c\left(x, y_{0}\right)$ for some $b$-concave $v: Z \rightarrow \mathbb{R}$. By [23], we know that if (A3w') fails, then the set of $C^{1}, b$-concave functions is not dense in the set of all $b$-concave functions in the $L^{\infty}(Z)$ topology. From this it follows easily that the set of $C^{1}, c$-concave functions is not dense in the set of all $c$-concave functions in the $L^{\infty}(X)$ topology. The argument in [23] now implies the existence of smooth marginals $\mu$ and $\nu$ and a $c$-concave $u=v(Q(x))+c\left(x, y_{0}\right)$ which is not $C^{1}$, such that the optimal map is of the form $F(x)=c-\exp _{x}(D u(x))$. As $u$ is not $C^{1}, v$ must not be $C^{1}$, and so the optimal map $T=b$ - $\exp _{z}(D v(z))$ between $\alpha=Q_{\#} \mu$ and $\nu$ is not continuous. It now follows that $F=T \circ Q$, is discontinuous.

\section{Regularity for non-c-convex targets}

The results in the previous section imply that we cannot hope that the optimizer is continuous for arbitrary smooth data if the cost is not reducible. It is then natural to ask for which marginals can we expect the optimal map to be smooth? In this section, we study this question in the special case when $n=1$. We identify conditions on the interaction between the marginals and the cost that allow us to find an explicit formula for the optimal map and prove that it is continuous.

We will assume $Y=(a, b) \subset \mathbb{R}$ is an open interval and that $X$ is a bounded domain in $\mathbb{R}^{m}$. We will also assume that $c \in C^{2}(\bar{X} \times \bar{Y})$ satisfies (A2'), which in this setting simply means that the gradient $\nabla_{x}\left(\frac{\partial c}{\partial y}\right)$ never vanishes, and that the level sets $L_{x}(y)$ are all connected. Therefore, these sets will all be $C^{1}$ submanifolds of dimension $m-1$. We define the following set:

$$
\begin{aligned}
P=\{\tilde{x} \in \bar{X}: \quad & \forall y_{0}, y_{1} \in \bar{Y}, x \in L_{\tilde{x}}\left(y_{0}\right), \text { we have } \frac{\partial c\left(\tilde{x}, y_{1}\right)}{\partial y} \leq \frac{\partial c\left(x, y_{1}\right)}{\partial y} \\
& \text { if } \left.y_{0}<y_{1}, \text { and } \frac{\partial c\left(\tilde{x}, y_{1}\right)}{\partial y} \geq \frac{\partial c\left(x, y_{1}\right)}{\partial y} \text { if } y_{0}>y_{1}\right\}
\end{aligned}
$$

\footnotetext{
${ }^{1}$ It should be noted that the while the boundary of $X$ is not smooth here, this is not the reason for the discontinuity in $h$; the corners of the boundary can be mollified and the density will still be discontinuous at 0 .
} 
When the level sets $L_{x}(y)$ are independent of $y, P$ is the entire domain $X$. If not, $P$ consists of points $\tilde{x}$ for which the level sets $L_{\tilde{x}}(y)$ evolve with $y$ in a monotonic way. The submanifold $L_{\tilde{x}}\left(y_{1}\right)$ divides the region $X$ into two subregions: $\left\{x: \frac{\partial c\left(\tilde{x}, y_{1}\right)}{\partial y}>\frac{\partial c\left(x, y_{1}\right)}{\partial y}\right\}$ and $\left\{x: \frac{\partial c\left(\tilde{x}, y_{1}\right)}{\partial y} \leq \frac{\partial c\left(x, y_{1}\right)}{\partial y}\right\}$. The condition $\tilde{x} \in P$ ensures that for $y_{0}<y_{1}$, the set $L_{\tilde{x}}\left(y_{0}\right)$ will lie entirely in the latter region. For interior points, the submanifolds $L_{\tilde{x}}\left(y_{0}\right)$ and $L_{\tilde{x}}\left(y_{1}\right)$ will generically intersect transversely and so $L_{\tilde{x}}\left(y_{0}\right)$ will intersect both of these regions; therefore, interior points will typically not belong to $P$. On the other hand, if $\tilde{x}$ is a boundary point and $m=2$, we can heuristically view the level sets $L_{\tilde{x}}(y)$ (curves in this case) as rotating about the point $\tilde{x}$; if this rotation is always in a particular, fixed direction, then $\tilde{x}$ is in $P$.

We now state two definitions, followed by the main result of this section.

Definition 4.1. We say $y$ splits the mass at $x$ if

$$
\mu\left(\left\{\bar{x}: \frac{\partial c(x, y)}{\partial y}<\frac{\partial c(\bar{x}, y)}{\partial y}\right\}\right)=\nu([a, y))
$$

If $\mu$ and $\nu$ are absolutely continuous with respect to Lebesgue measure, this is equivalent to

$$
\mu\left(\left\{\bar{x}: \frac{\partial c(x, y)}{\partial y}>\frac{\partial c(\bar{x}, y)}{\partial y}\right\}\right)=\nu([y, b]) .
$$

Definition 4.2. Let $\tilde{x} \in P$. We say $\tilde{x}$ satisfies the mass comparison property (MCP) if for all $y_{0}<y_{1} \in \bar{Y}$ we have

$$
\mu\left(\bigcup_{y \in\left[y_{0}, y_{1}\right]} L_{\tilde{x}}(y)\right)<\nu\left(\left[y_{0}, y_{1}\right]\right)
$$

In the case when the level sets $L_{x}(y)$ are independent of $y$, the MCP is satisfied for all $x \in P=\bar{X}$ as long as $\mu$ assigns zero mass to every $L_{x}(y)$ and $\nu$ assigns non-zero mass to every open interval. Alternatively, in view of the previous section, we know that in this case the cost has the form $c(Q(x), y)$, where $Q: X \rightarrow Z$ and $Z=\left[z_{0}, z_{1}\right] \subseteq \mathbb{R}$ is an interval; the MCP boils down to the assumption that $\alpha=Q_{\#} \mu$ assigns zero mass to all singletons and $\nu$ assigns non-zero mass to every open interval.

We are now ready to state the main result of this section. In what follows, $\gamma$ will be a solution to the Kantorovich problem. The support of $\gamma$, or $\operatorname{spt}(\gamma)$, is the smallest closed subset of $\bar{X} \times \bar{Y}$ of full mass.

Theorem 4.1. Suppose $\mu$ and $\nu$ are absolutely continuous with respect to Lebesgue. Suppose that for all $x, y \in \bar{X} \times \bar{Y}$ such that $y$ splits the mass at $x$ there exists an $\tilde{x} \in P \cap L_{x}(y)$ satisfying the $M C P$. Then for each $x \in \bar{X}$ there is a unique $y \in \bar{Y}$ that splits the mass at $x$. Moreover, $(x, y) \in \operatorname{spt}(\gamma)$ and $(x, \bar{y}) \notin \operatorname{spt}(\gamma)$ for all other $\bar{y} \in \bar{Y}$. Therefore, the optimal map is well defined everywhere.

Note that we can actually use Theorem 4.1 to derive a formula for the optimal map:

$$
F(x):=\sup \left\{y: \mu\left(\left\{\bar{x}: \frac{\partial c(x, y)}{\partial y}<\frac{\partial c(\bar{x}, y)}{\partial y}\right\}\right)>\nu([a, y))\right\}
$$


Most of this section will be devoted to proving Theorem 4.1. Before we launch into the details of the proof, however, we will briefly discuss its implications. We first show that Theorem 4.1 immediately implies the following corollary on the continuity of the optimizer. We then illustrate the corollary with two simple examples. In the first example, the conditions of Theorem 4.1 are violated and we demonstrate explicitly that the optimal map is discontinuous. In the second, the conditions are satisfied and so Theorem 4.1 implies that the optimal map is continuous.

Corollary 4.1. Under the assumptions of the preceding theorem, the optimal map is continuous on $\bar{X}$.

Proof. Choose $x_{k} \rightarrow x \in \bar{X}$ and set $y_{k}=F\left(x_{k}\right)$; we need to show $y_{k} \rightarrow F(x)$. Set $\bar{y}=\limsup _{k \rightarrow \infty} y_{k} \in \bar{Y}$; by passing to a subsequence we can assume $y_{k} \rightarrow \bar{y}$. As $\operatorname{spt}(\gamma)$ is closed by definition, we must have $(x, \bar{y}) \in \operatorname{spt}(\gamma)$ and so Theorem 4.1 implies $\bar{y}=F(x)$. A similar argument implies $\liminf _{k \rightarrow \infty} y_{k}=F(x)$, completing the proof.

Example 4.1. Let $X$ be the quarter disk in $\mathbb{R}^{2}$ :

$$
X=\left\{\left(x_{1}, x_{2}\right): x_{1}>0, x_{2}>0, x_{1}^{2}+x_{2}^{2}<1\right\} .
$$

Let $Y=\left(0, \frac{\pi}{2}\right)$ and take $\mu$ and $\nu$ to be uniform measures on $X$ and $Y$, respectively, scaled so that both have total mass 1 . Let $c(x, y)=-x_{1} \cos (y)-x_{2} \sin (y)$; this is equivalent to the Euclidean distance between $x$ and the point on the unit circle parameterized by the polar angle $y$. We claim that the optimal map takes the form $F(x)=\arctan \left(\frac{x_{2}}{x_{1}}\right)$; that is, each point $x$ is mapped to the point $\frac{x}{|x|}$ on the unit circle. Indeed, note that

$$
c(x, y) \geq-\sqrt{x_{1}^{2}+x_{2}^{2}}
$$

with equality if and only if $y=F(x)$, and that uniform measure on the graph $(x, F(x))$ projects to $\mu$ and $\nu$, implying the desired result. Now observe that $F$ is discontinuous at $(0,0)$; in fact, $((0,0), y)$ satisfies $(4.1)$ for all $y \in Y$ so the optimal measure pairs the origin with every point. We now demonstrate explicitly that the conditions of Theorem 4.1 fail in this case. Choose any $y$ and set $x=(0,0)$; then, as $\frac{\partial c(x, y)}{\partial y}=$ $x_{1} \sin (y)-x_{2} \cos (y)$, the level set $L_{(0,0)}(y)$ is the straight line segment passing through $(0,0)$ and the point on the unit circle with polar angle $y$. As $\mu$ and $\nu$ are uniform, this means that $y$ splits the mass at $(0,0)$; the main condition in the theorem is that there exists some $\tilde{x} \in P \cap L_{(0,0)}(y)$ satisfying the MCP.

We now show that no such $\tilde{x}$ exists. First note that $y \mapsto \frac{\partial c(x, y)}{\partial y}=x_{1} \sin (y)-$ $x_{2} \cos (y)$ is strictly increasing, then for $y_{1}>y_{0}$ and any $x \in L_{0,0)}\left(y_{0}\right)$ we have

$$
\frac{\partial c\left(x, y_{1}\right)}{\partial y}>\frac{\partial c\left(x, y_{0}\right)}{\partial y}=\frac{\partial c\left((0,0), y_{0}\right)}{\partial y}=0=\frac{\partial c\left((0,0), y_{1}\right)}{\partial y}
$$

This implies that $\tilde{x}=(0,0) \in P$; a similar calculation implies that $x \notin P$ for all other $x \in L_{(0,0)}(y)$, so that $P \cap L_{(0,0)}(y)=\tilde{x}$. But $\tilde{x}$ does not satisfy the MCP; indeed, as $\mu$ and $\nu$ are uniform, the characterization of the sets $L_{(0,0)}(y)$ described 
above implies that, for $y_{1} \geq y_{0}$,

$$
\mu\left(\bigcup_{y \in\left[y_{0}, y_{1}\right]} L_{\tilde{x}}(y)\right)=\nu\left(\left[y_{0}, y_{1}\right]\right) .
$$

Example 4.2. Take $X, Y, c$ and $\mu$ to be as in the last example, but now suppose that $\nu$ is uniform measure on $\left(0, \frac{\pi}{4}\right)$, rescaled to have total mass 1 . It is not hard to check that $\left(0, x_{2}\right)$ is in $P$ and satisfies the MCP for all $x_{2}$. Now, for all $(x, y) \in \bar{X} \times \bar{Y}$ such that $y$ splits the mass at $x$, it is straightforward to verify that we have some $\left(0, x_{2}\right) \in L_{x}(y)$; hence, Corollary 4.1 implies continuity of the optimizer.

The proof of Theorem 4.1 requires some additional machinery, which we begin to build up now.

Lemma 4.1. Suppose $\tilde{x} \in P, x \in \bar{X}, y_{0}, y_{1} \in \bar{Y}$ and $\left(\tilde{x}, y_{1}\right),\left(x, y_{0}\right) \in \operatorname{spt}(\gamma)$. Then $\frac{\partial c\left(x, y_{1}\right)}{\partial y} \geq \frac{\partial c\left(\tilde{x}, y_{1}\right)}{\partial y}$ if $y_{0}<y_{1}$ and $\frac{\partial c\left(x, y_{1}\right)}{\partial y} \leq \frac{\partial c\left(\tilde{x}, y_{1}\right)}{\partial y}$ if $y_{0}>y_{1}$.

Proof. The support of $\gamma$ is $c$-monotone (see [32] for a proof); this means that $c\left(\tilde{x}, y_{1}\right)+$ $c\left(x, y_{0}\right) \leq c\left(\tilde{x}, y_{0}\right)+c\left(x, y_{1}\right)$. If $y_{0}<y_{1}$, this implies

$$
\int_{y_{0}}^{y_{1}} \frac{\partial c(\tilde{x}, y)}{\partial y} d y \leq \int_{y_{0}}^{y_{1}} \frac{\partial c(x, y)}{\partial y} d y .
$$

This means that there is some $y \in\left[y_{0}, y_{1}\right]$ such that $\frac{\partial c(\tilde{x}, y)}{\partial y} \leq \frac{\partial c(x, y)}{\partial y}$; if we had $\frac{\partial c\left(\tilde{x}, y_{1}\right)}{\partial y}>\frac{\partial c\left(x, y_{1}\right)}{\partial y}$, the Intermediate Value Theorem would imply the existence of a $\bar{y} \in$ $\left(y, y_{1}\right)$ such that $\frac{\partial c(\tilde{x}, \bar{y})}{\partial y}=\frac{\partial c(x, \bar{y})}{\partial y}$, or $x \in L_{\tilde{x}}(\bar{y})$. This, together with our assumption $\frac{\partial c\left(\tilde{x}, y_{1}\right)}{\partial y}>\frac{\partial c\left(x, y_{1}\right)}{\partial y}$, violates the condition $\tilde{x} \in P$; we conclude that $\frac{\partial c\left(\tilde{x}, y_{1}\right)}{\partial y} \leq \frac{\partial c\left(x, y_{1}\right)}{\partial y}$ A similar argument shows $\frac{\partial c\left(\tilde{x}, y_{1}\right)}{\partial y} \geq \frac{\partial c\left(x, y_{1}\right)}{\partial y}$ if $y_{0}>y_{1}$.

Lemma 4.1 immediately implies the following.

Lemma 4.2. Suppose $\mu$ and $\nu$ are absolutely continuous with respect to Lebesgue measure. Then if $\tilde{x} \in P, y \in \bar{Y}$ and $(\tilde{x}, y) \in \operatorname{spt}(\gamma), y$ splits the mass at $\tilde{x}$.

Lemma 4.3. Suppose $\mu$ and $\nu$ are absolutely continuous with respect to Lebesgue. Then, for each $x \in \bar{X}$ there is a $y \in \bar{Y}$ that splits the mass at $x$.

Proof. The function $y \mapsto f_{x}(y):=\mu\left(\left\{\bar{x}: \frac{\partial c(x, y)}{\partial y}<\frac{\partial c(\bar{x}, y)}{\partial y}\right\}\right)-\nu([a, y))$ is continuous. Observe that $f_{x}(a) \geq 0$ and $f_{x}(b) \leq 0$; the result now follows from the Intermediate Value Theorem.

Similarly, it is straightforward to prove the following lemma.

Lemma 4.4. Suppose $\mu$ and $\nu$ are absolutely continuous with respect to Lebesgue. Then, for each $y \in \bar{Y}$ there is an $x \in X$ such that $y$ splits the mass at $\bar{x}$ if and only if $\bar{x} \in L_{x}(y)$.

Lemma 4.5. Suppose $\mu$ and $\nu$ are absolutely continuous with respect to Lebesgue measure and that $\tilde{x} \in P$ satisfies the $M C P$. Then there is a unique $y \in \bar{Y}$ that splits the mass at $\tilde{x}$. 
Proof. Existence follows from Lemma 4.3; we must only show uniqueness. Our proof is by contradiction; suppose $y_{0}<y_{1} \in \bar{Y}$ both split the mass at $\tilde{x}$. For any $x$ such that $\frac{\partial c\left(x, y_{0}\right)}{\partial y}<\frac{\partial c\left(\tilde{x}, y_{0}\right)}{\partial y}$ and $\frac{\partial c\left(x, y_{1}\right)}{\partial y}>\frac{\partial c\left(\tilde{x}, y_{1}\right)}{\partial y}$ the Intermediate Value Theorem yields a $y \in\left[y_{0}, y_{1}\right]$ such that $x \in L_{\tilde{x}}(y)$; hence, setting $X_{0}=\left\{x: \frac{\partial c\left(x, y_{0}\right)}{\partial y}<\frac{\partial c\left(\tilde{x}, y_{0}\right)}{\partial y}\right\}$ and $X_{1}=\left\{x: \frac{\partial c\left(x, y_{1}\right)}{\partial y}>\frac{\partial c\left(\tilde{x}, y_{1}\right)}{\partial y}\right\}$, we have

$$
X_{0} \bigcap X_{1} \subseteq \bigcup_{y \in\left[y_{0}, y_{1}\right]} L_{\tilde{x}}(y) .
$$

Therefore

$$
\mu\left(X_{0} \bigcap X_{1}\right) \leq \mu\left(\bigcup_{y \in\left[y_{0}, y_{1}\right]} L_{\tilde{x}}(y)\right)
$$

Now, using absolute continuity of $\mu$ and $\nu$ together with the assumption that $y_{0}$ and $y_{1}$ split the mass at $\tilde{x}$, we have

$$
\begin{aligned}
\mu\left(X_{0} \cap X_{1}\right)=\mu\left(X_{0}\right)+\mu\left(X_{1}\right)-\mu\left(X_{0} \cup X_{1}\right) & \geq \mu\left(X_{0}\right)+\mu\left(X_{1}\right)-1 \\
& =\nu\left(\left[y_{0}, b\right]\right)+\nu\left(\left[a, y_{1}\right]\right)-1 \\
& =1-\nu\left(\left[a, y_{0}\right]\right)+1-\nu\left(\left[y_{1}, b\right]\right)-1 \\
& =1-\nu\left(\left[a, y_{0}\right]\right)-\nu\left(\left[y_{1}, b\right]\right) \\
& =\nu\left(\left[y_{0}, y_{1}\right]\right) .
\end{aligned}
$$

Combining (4.3), (4.4) and the MCP now yields a contradiction.

We are now ready to prove Theorem 4.1.

Proof. For each $x \in \bar{X}$, by Lemma 4.3 we can choose $y \in \bar{Y}$ that splits the mass at $x$; the hypothesis then implies the existence of $\tilde{x} \in P \cap L_{x}(y)$ satisfying the MCP. As we must have $(\tilde{x}, \tilde{y}) \in \operatorname{spt}(\gamma)$ for some $\tilde{y}$, Lemmas 4.5 and 4.2 imply that $(\tilde{x}, y) \in \operatorname{spt}(\gamma)$.

We now show that

$$
\left(x, y^{\prime}\right) \notin \operatorname{spt}(\gamma), \text { for all } y^{\prime} \neq y
$$

The proof is by contradiction; to this end, assume $\left(x, y^{\prime}\right) \in \operatorname{spt}(\gamma)$ for some $y^{\prime} \neq y$. Suppose $y^{\prime}>y$; choose $\bar{y} \in\left(y, y^{\prime}\right)$. By Lemma 4 .4, we can choose $\bar{x}$ such that $\bar{y}$ splits the mass at $\bar{x}$. Now use the hypothesis of the theorem again to find $\tilde{\tilde{x}} \in P \cap L_{\bar{x}}(\bar{y})$ satisfying the MCP and note that $(\tilde{\tilde{x}}, \bar{y}) \in \operatorname{spt}(\gamma)$. Now, as $y$ splits the mass at $\tilde{x}, \bar{y}$ does not split the mass at $\tilde{x}$ by Lemma 4.5 and so $\tilde{x} \notin L_{\tilde{\tilde{x}}}(\bar{y})$ (as $\bar{y}$ splits the mass at $\tilde{\tilde{x}})$. This means that $\frac{\partial c(\tilde{x}, \bar{y})}{\partial y} \neq \frac{\partial c(\tilde{\tilde{x}}, \bar{y})}{\partial y}$; Lemma 4.1 now implies $\frac{\partial c(\tilde{x}, \bar{y})}{\partial y}>\frac{\partial c(\tilde{\tilde{x}}, \bar{y})}{\partial y}$.

Therefore, as $\tilde{x} \in P$ and $x \in L_{\tilde{x}}(y)$,

$$
\begin{aligned}
\frac{\partial c(x, \bar{y})}{\partial y} & \geq \frac{\partial c(\tilde{x}, \bar{y})}{\partial y} \\
& >\frac{\partial c(\tilde{\tilde{x}}, \bar{y})}{\partial y}
\end{aligned}
$$

But now $\left(x, y^{\prime}\right),(\tilde{\tilde{x}}, \bar{y}) \in \operatorname{spt}(\gamma)$ and $y^{\prime}>\bar{y}$ contradicts Lemma 4.1. An analogous argument implies that we cannot have $\left(x, y^{\prime}\right) \in \operatorname{spt}(\gamma)$ for $y^{\prime}<y$, completing the proof of (4.5). 
Now, note that we must have $(x, \bar{y}) \in \operatorname{spt}(\gamma)$ for some $\bar{y} \in Y$ and so the preceding argument implies $(x, y) \in \operatorname{spt}(\gamma)$.

Finally, we must show that there is no other $y^{\prime} \in \bar{Y}$ which splits the mass at $x$; this follows immediately, as if there were such a $y^{\prime}$, an argument analogous to the preceding one would imply that $\left(x, y^{\prime}\right) \in \operatorname{spt}(\gamma)$, contradicting (4.5).

\section{Acknowledgments}

The author was supported in part by an NSERC postgraduate scholarship. This work was completed in partial fulfillment of the requirements of a doctoral degree in mathematics at the University of Toronto. The author is pleased to thank Robert McCann and Paul Lee for fruitful discussions during the course of this work. He is also grateful to an anonymous referee for many useful comments and suggestions.

\section{References}

[1] S. Basov, Hamiltonian approach to multi-dimensional screening, J. Math. Econom. 36 (2001), 77-94.

[2] Y. Brenier, Decomposition polaire et rearrangement monotone des champs de vecteurs, C. R. Acad. Sci. Pair. Ser. I Math. 305 (1987), 805-808.

[3] L.A. Caffarelli, The regularity of mappings with a convex potential, J. Amer. Math. Soc. 5 (1992), 99-104.

[4] L.A. Caffarelli, Boundary regularity of maps with convex potentials, Comm. Pure Appl. Math. 45 (1992), 1141-1151.

[5] L.A. Caffarelli, Boundary regularity of maps with convex potentials-II, Ann. of Math. (2) 144 (1996), 453-496.

[6] L. Caffarelli, Allocation maps with general cost functions, in 'Partial differential equations and applications' (P. Marcellini, G. Talenti and E. Vesintini, eds.), Lecture Notes in Pure Appl. Math., 177, 1996, 29-35.

[7] P. Castillon, Submanifolds, isoperimetric inequalities and optimal transportation, J. Funct. Anal. 259 (2010), 79-103.

[8] P. Delanoë, Classical solvability in dimension two of the second boundary-value problem associated with the Monge-Ampere operator, Ann. Inst. H. Poincare Anal. Non Lineaire 8 (1991), 442-457.

[9] P. Delanoë, Gradient rearrangement for diffeomorphisms of a compact manifold, Differential Geom. Appl. 20 (2004), 145-165.

[10] R. Deneckere and S. Severinov, Multi-dimensional screening with a one dimensional allocation space, preprint.

[11] A. Figalli, Y.-H. Kim and R.J. McCann, Holder continuity and injectivity of optimal maps, http://arxiv.org/abs/1107.1014v1.

[12] A. Figalli, L. Rifford and C. Villani, Necessary and sufficient conditions for continuity of optimal transport maps on Riemannian manifolds, Tohoku Math. J. (2), 63(4) (2011), 855-876.

[13] A. Figalli and L. Rifford, Continuity of optimal transport maps on small deformations of $\mathbb{S}^{2}$, Comm. Pure Appl. Math. 62(12) (2009), 1670-1706.

[14] A. Figalli, L. Rifford and C. Villani, Necessary and sufficient conditions for continuity of optimal transport maps on Riemannian manifolds, Tohoku Math. J., to appear.

[15] A. Figalli, L. Rifford and C. Villani, Nearly round spheres look convex, Amer. J. Math. 134(1) (2012), 109-139.

[16] W. Gangbo, Habilitation thesis, Universite de Metz, 1995.

[17] W. Gangbo and R.J. McCann, The geometry of optimal transportation, Acta Math. 177 (1996), $113-161$.

[18] Y.-H. Kim, Counterexamples to continuity of optimal transportation on positively curved Riemannian manifolds, Int. Math. Res. Not. 2008 (2008), article ID rnn120, 15 pages, doi:10.1093/imrn/rnn120. 
[19] Y-H. Kim and R.J. McCann, Continuity, curvature and the general covariance of optimal transportation, J. Eur. Math. Soc. 12 (2010), 1009-1040.

[20] Y-H. Kim and R.J. McCann, Towards the smoothness of optimal maps on Riemannian submersions and Riemannian products (of round spheres in particular), J. Reine Angew. Math. 2012 (2012), 1-27.

[21] V. Levin, Abstract cyclical monotonicity and Monge solutions for the general MongeKantorovich problem, Set-Valued Anal. 7(1) (1999), 7-32.

[22] J. Liu, Hölder regularity in optimal mappings in optimal transportation, Calc. Var. Partial Differential Equations 41 (2011), 285-299.

[23] G. Loeper, On the regularity of maps solutions of optimal transportation problems, Acta Math. 202 (2009), 241-283.

[24] G. Loeper, Regularity of optimal maps on the sphere: the quadratic cost and the reflector antenna, Arch. Ration. Mech. Anal. 199(1) (2011), 269-289.

[25] G. Loeper and C. Villani, Regularity of optimal transport in curved geometry: the nonfocal case, Duke Math. J. 151(3) (2010), 431-485.

[26] X-N. Ma, N. Trudinger and X-J. Wang, Regularity of potential functions of the optimal transportation problem, Arch. Ration. Mech. Anal. 177 (2005), 151-183.

[27] R.J. McCann, Polar factorization of maps on Riemannian manifolds, Geom. Funct. Anal. 11 (2001), 589-608.

[28] R. McCann, B. Pass and M. Warren, Rectifiability of optimal transportation plans, Canad. J. Math., http://dx.doi.org/10.4153/CJM-2011-080-6.

[29] B. Pass, On the local structure of optimal measures in the multi-marginal optimal transportation problem, Calc. Var. and PDE. 43 (2012), 529-536.

[30] B. Pass, Ph.D. thesis, University of Toronto, 2011.

[31] B. Pass, Convexity and multi-dimensional screening for spaces with different dimensions, J. Econom. Theory, to appear, http://dx.doi.org/10.1016/j.jet.2012.05.004.

[32] C. Smith and M. Knott, On Hoeffding-Frechet bounds and cyclic monotone relations, J. Multivariate Anal. 40 (1992), 328-334.

[33] N. Trudinger and X.-J. Wang, On the second boundary value problem for Monge-Ampere type equations and optimal transportation, Ann. Sc. Norm. Super. Pisa Cl. Sci. 8(1) (2009), 143-174.

[34] N. Trudinger and X.-J. Wang, On strict convexity and $C^{1}$-regularity of potential functions in optimal transportation, Arch. Ration. Mech. Anal. 192(3) (2009), 403-418.

[35] J. Urbas, On the second boundary value problem for equations of Monge-Ampere type, J. Reine Angew. Math. 487 (1997), 115-124.

[36] X-J. Wang, On the design of a reflector antenna, Inverse Problems 12(3) (1996), 351-375.

Department of Mathematics, University of Toronto, Toronto, ON M5S 2E3, Canada

Current address: Department of Mathematical and Statistical Sciences, University of

Alberta, Edmonton, AB T6G 2G1, Canada

E-mail address: pass@ualberta.ca

$U R L$ : www.ualberta.ca/pass 
